Egyptian

Orthodontic Journal

\title{
REACTION OF PERIODONTIUM TO ORTHODONTIC FORCE IN DIABETIC RATS
}

\author{
Ahmed A El-bialy ${ }^{\star}$ and Yousry M El-Hawary ${ }^{\star *}$
}

\section{ABSTRACT}

This study was carried-out to evaluate the reaction of the periodontiume to orthodontic force in diabetic rats and to detect the nitric oxide with its tissue response during bone remodeling. The sample consisted of eighteen male albino rats at the beginning of the study and ended by sixteen rats due to sample decay. The sample was divided into two equal groups. The first group (8 rats) was used as non-diabetic controls. The second group (8 rats) served as the diabetic group and was treated by single intraperitoneal injection of Streptozotocin $(S T Z)$ with $50 \mathrm{Mg} / \mathrm{Kg}$ dissolved in citrate-6uffered saline solution, $(p h=4.5)^{(11)}$.

The orthodontic treatment was tried in many ways; a spring (one ounce force) was stretched between the two mandibular incisors to elicit expansion. The spring was designed in such a way that a helix was constructed in a single vertical loop and attached to the teeth by eyelets ${ }^{(14)}$. Then, different sizes of elastic separators retented by kobayashi ligatures. The specimens were taken, washed, fixed in fixative solution, put in EDTA for decalcification and after that prepared until reached to paraffin embedding. Specimens stained by haematoxilin and eosin(15), Mason's trichrome(16). And then the immunohistochemical staining of $\mathcal{N O S}^{(17,18)}$.

The diabetic group showed greater numbers of osteoclast cells in their lacuna indicating more bone resorption compared to non diabetic rats. In spite of highly expression of the $\mathcal{N O}$ in the pressure side of the

\footnotetext{
* Associate Professor Orthodontics, Faculty of Dentistry, Mansoura University, Egypt.

** Lecturer of Oral biology, Faculty of Dentistry, Mansoura University, Egypt.
} 
Egyptian

Orthodontic Journal

diabetic group, it was founded with low reaction in the tension side. Depending on the results of this study, the diabetic rats showed greater bone resorption at the pressure side and retarded bone deposition at the tension side compared to the healthy rats. Hence, orthodontist should consider these results by using a very light force, increasing the gaps between visits, planning to decrease the range of tooth movement, monitoring the oral hygiene and sharing with patients physicians for controlling diabetes during orthodontic managements.

\section{INTRODUCTION}

Diabetes mellitus (DM) is diagnosed in $3 \%$ to $4 \%$ of the population ${ }^{(1)}$. It compromises two main types: type 1 with a total deficiency in insulin secretion, type 2 is a combination of resistance to insulin action and inadequate compensatory insulin secretion. However, more adults are now likely to seek for orthodontic treatment. When diabetes mellitus (DM) is a part of patient history, the orthodontist should consider its consequences to the treatment. Holtgrave and Donath ${ }^{(2)}$ during their study found that there was retarded osseous regeneration, a weakening of the periodontal ligament, and microangiopathes in the gingival area. The diabetic patients especially type 2 should be considered during orthodontic treatment planning.

It has been shown that nitric oxide (NO) synthesis can be induced in osteoblast and osteocytes by any mechanical stress or strain. Many investigators ${ }^{(3,4)}$ have been showed that osteocytes showed greater increase in NO production as a result of mechanical loadings than osteoblast. Their results supported the hypothesis that osteocytes are the principal sensors and effectors of mechanical stress in bone ${ }^{(5)}$. Studies of Shirazi et $\mathrm{al}^{(6)}$ and Wennstrom et $\mathrm{al}^{(7-9)}$ showed that different factors might influence the rate of orthodontic tooth movement via various biomediators, and NO is the major important local factor that regulates bone metabolism.

Nitric oxide (No) is an important regulatory molecule in bone formation and resorption. It is involved in second messenger formation, osteoblast and osteoclast function. It is suggested that it has an important biochemical mediator in the response of the periodontal tissue to orthodontic force and it is produced primarily through the activity of constitutive Nitric oxide synthase (NOS) ${ }^{(10)}$. This study was carried-out to evaluate the reaction of the Periodontium to orthodontic force in diabetic rats and to detect the nitric oxide with its tissue response during bone remodeling. 
Egyptian

Orthodontic Journal

\section{Material and Methods:}

The sample consisted of eighteen male albino rats at the beginning of the study and ended by sixteen rats due to sample decay. The rats were purchased from animal house, Faculty of science, Mansoura University. Female rats were excluded due to the short menstruation period and the hormonal disturbance.

The sample was divided into two equal groups. The first group (8 rats) was used as non-diabetic controls. The second group (8 rats) served as the diabetic group and was treated by single intraperitoneal injection of Streptozotocin*(STZ) with 50 $\mathrm{Mg} / \mathrm{Kg}$ dissolved in citrate-buffered saline solution, $(\mathrm{ph}=4.5)^{(11)}$. Induction of diabetes was attained when a fasting blood glucose level was at least double the baseline value and this was achieved on the third day after the injection ${ }^{(12)}$.

\section{Daily care:}

Individual cages were used for animal housing. Soft diet was used to prevent appliance breakdown by hard type of food. Fluid was administrated as needed depending upon the specialist supervision. The initial average weight was $180 \pm 18$ grams with 9 weeks old and the mean weight loss by the end of 14 days experimental period was $20 \mathrm{gm} \pm 3$ in the control group while it was $42 \pm 36$ in the diabetic group.

\section{Anesthesia:}

Each animal was anesthetized by Xylazine $1 \mathrm{mg} / \mathrm{kg}$ in conjunction with ketamine $10 \mathrm{mg} / \mathrm{kg}{ }^{(13)}$.

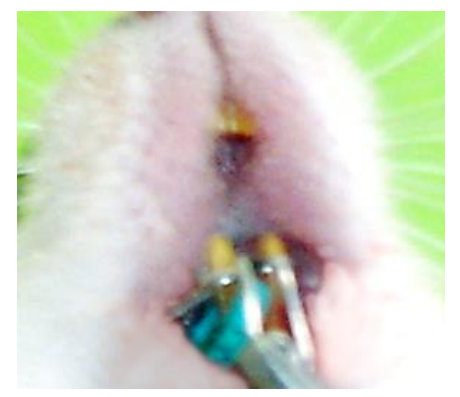

Fig. (1): Expansion Design

\section{Orthodontic treatment and measurements:}

The orthodontic treatment was tried in many ways; first, a spring (one ounce force) was stretched between the two mandibular incisors to elicit expansion. The spring was designed in such a way that a helix was constructed in a single vertical loop 
and attached to the teeth by eyelets However, it was slipped within two days ${ }^{(14)}$. Additional small groove was prepared on each mandibular incisor for more spring retention but it slipped again. So, The orthodontic force was reapplied by insertion of different sizes of separating elastics between the mandibular incisors and retained by ligation of a Kobayashi design in the previous prepared groove. This elastic separators were changed periodically with the same size in both groups (every four days).

The gap between the mesial corners of the mandibular incisors was measured directly by each investigator at the beginning of the experiment then, with the end and the average between the readings was recorded.

Animals were sacrificed by using large dose of anesthesia at the end of the experiment. The specimens were taken, washed, fixed in fixative solution, put in EDTA for decalcification and after that prepared until reached to paraffin embedding. All specimens were stained by haematoxilin and $\operatorname{eosin}^{(15)}$, Mason's trichrome ${ }^{(16)}$. Then, the immunohistochemical staining of NOS ${ }^{(17,18)}$. The quantitative evaluation of the immune reactions were performed by determination of the integrated optical density (IOD) changes by digital image analysis ${ }^{(19)}$.

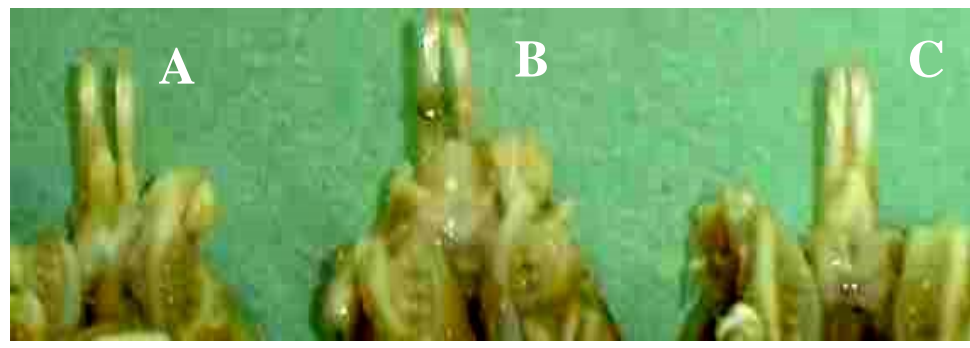

Fig.(2): Dissected mandibles showing tooth movement. A-Diabetic (after) b-Healthy (after) c- before expansion

\section{RESULTS}

Table 1: Means and standard deviations as well as the paired t value of the mean change of tooth movement in $\mathrm{mm}$.

\begin{tabular}{|c|c|c|c|c|c|c|}
\hline Variables & \multicolumn{2}{|c|}{ Non diabetic group } & \multicolumn{2}{|c|}{ diabetic group } & t-value & Significance \\
\hline Tooth movement & 6.32 & \pm 1.01 & 7.64 & \pm 1.72 & 6.34 & $0.0001 * * *$ \\
\hline
\end{tabular}


The specimens of the pressure side of diabetic group were stained with $\mathrm{H} \& \mathrm{E}$ shows large numbers of osteoclast in its deep howship's lacuna and more resorption of bone as in figure 4. On the other hand, the non diabetic group showed less numbers of osteoclast in shallow howship's lacuna and less resorption as in figure 3.

The same pressure side was stained by NO immunohistochemistry in diabetic group shows highly intense NO immunopositive reaction at the periodontal ligament and surface of the bone figure 10 in comparison to the non-diabetic group with less intensity than the diabetic group.

Immune reaction quantity by digital image shows the percentage in the periodontum of diabetic group was equal $13.7 \%$ figure 10 . But in non-diabetic it was $13.1 \%$ figure 9 . The specimens of tension side in diabetic group stained by Mason's trichrome stain shows less bone formation and some loss of architecture of the periodontal ligament fibers Figure 6. In comparison to non-diabetic group, it showed more bone formation and, good architecture of periodontal ligament figure 5 .

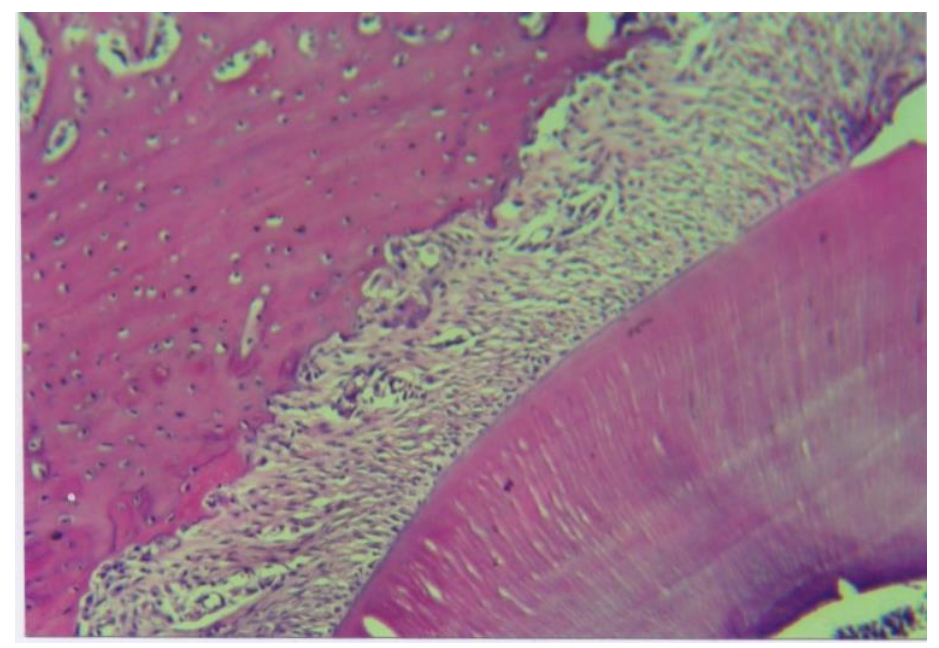

Fig. (3): Photomicrographic picture of Periodontium at the pressure side of nondiabetic group shows resorption of alveolar bone with less numbers of osteoclasts, widening of blood vessels and resorption of some areas in cementum (H\&E) stain. 


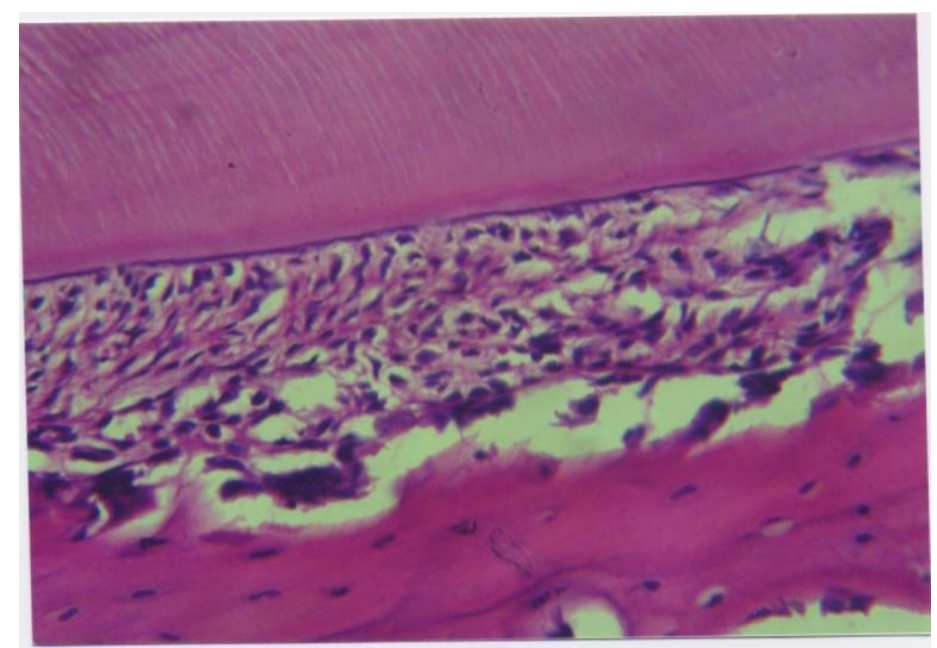

Fig. (4): Photomicrographic picture of the Periodontium at pressure side of diabetic group shows large numbers of osteoclasts in Howship's Lacuna, disfigurement of periodontal ligament and some area of resorption of cementum (H\&E) 400

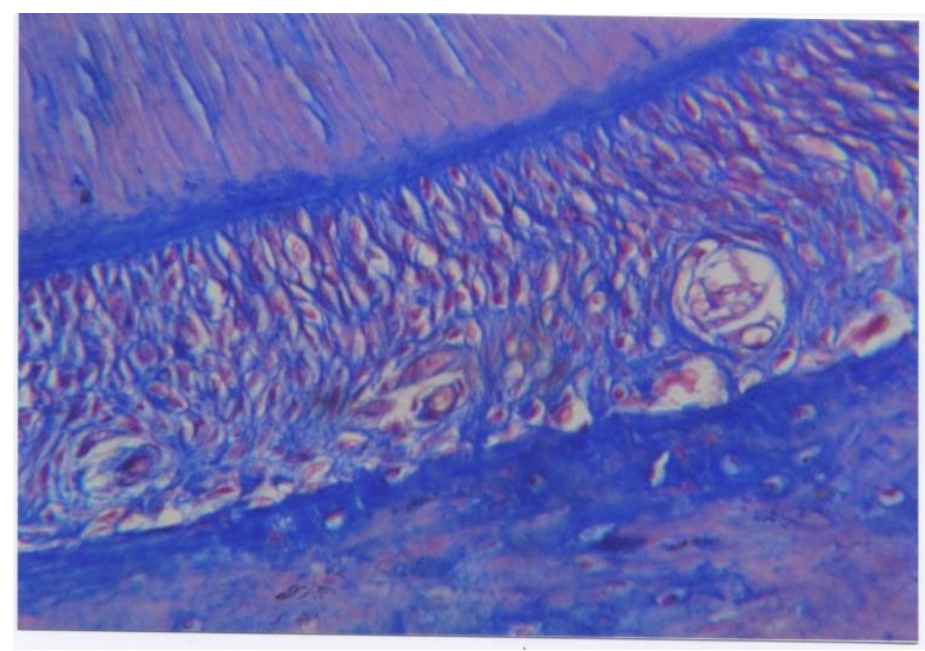

Fig. (5): Photomicrographic picture of nondiabetic group at tension side shows bone deposition at the surface of old bone ( Mason's trichrome stain). 


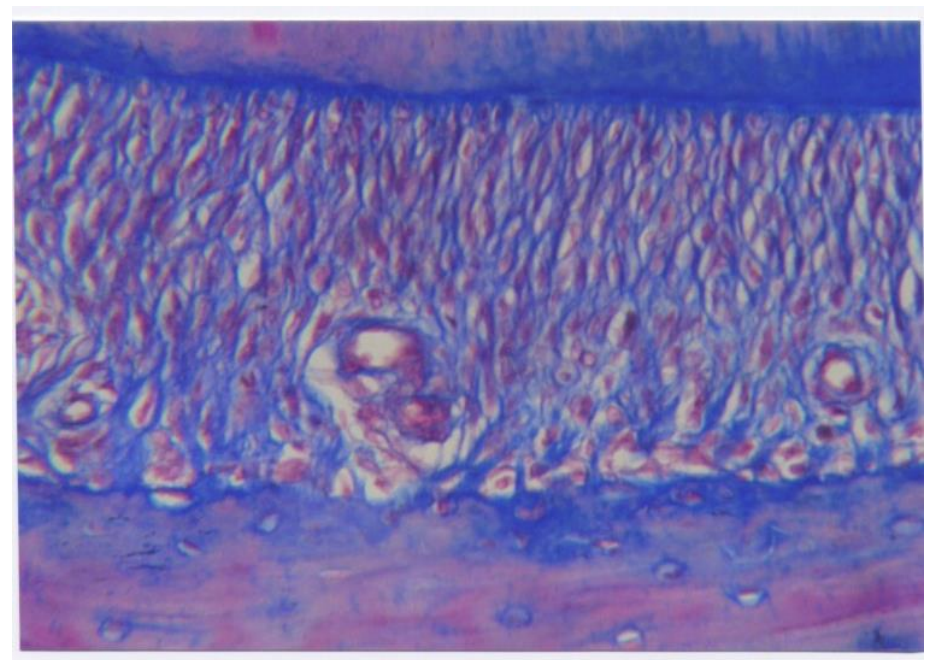

Fig. (6): Photomicrographic picture of diabetic group at tension side shows less bone deposition than nondiabetic group and slightly loss of architecture of periodontal ligament fibers ( Mason's trichrome stain).

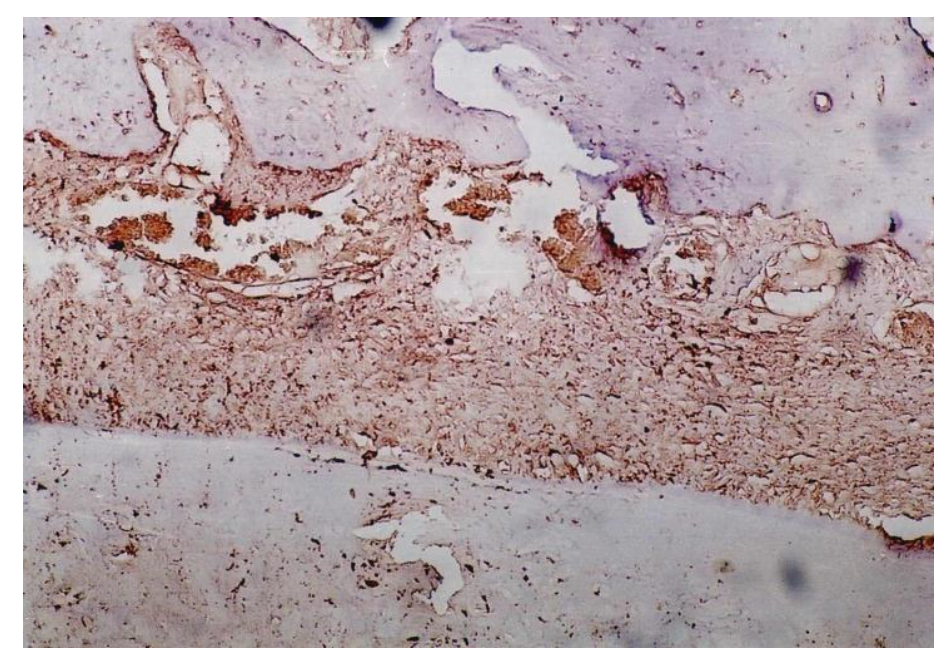

Fig. (7): Photomicrographic picture of nondiabetic group at pressure side shows intense immunopositive (N O) reaction related to blood vessels, surface of the bone, some areas of periodontal ligament and osteocytes but moderate reaction in other areas of periodontal ligament (immunohistochemical NO stain) 


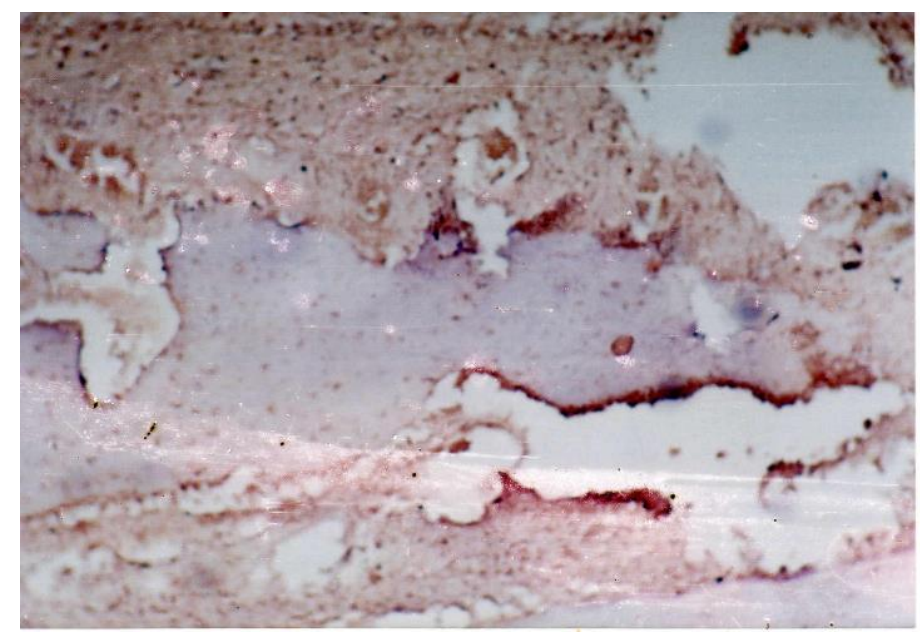

Fig.(8): Photomicrographic picture of diabetic group at pressure side shows highly intense immunopositive reaction in the periodontal ligament and at the surface of the bon but intense in other areas of periodontal ligament (immunohistochemical NO stain).

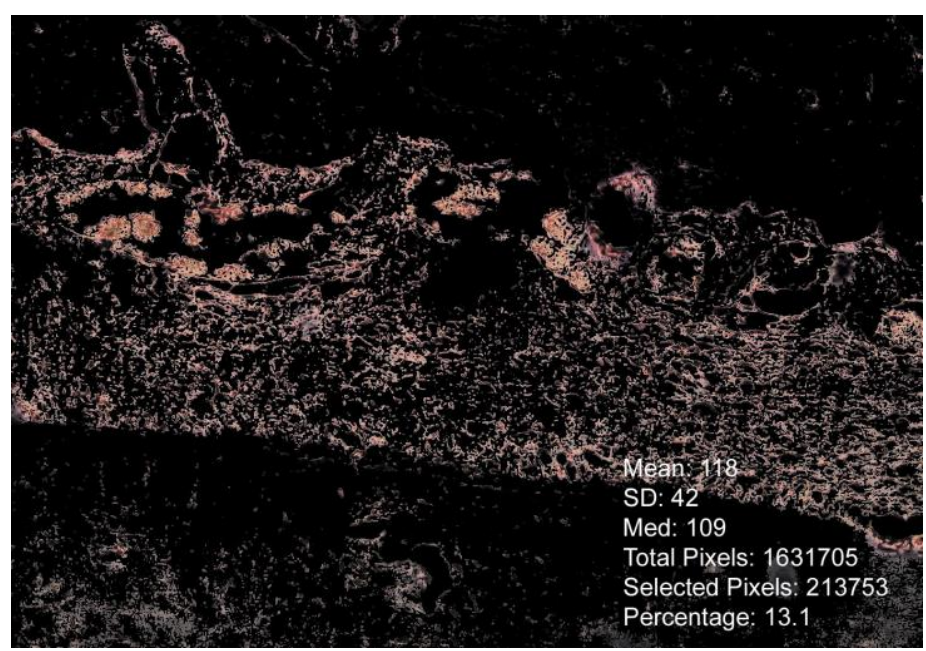

Fig.(9): digital image analysis of nondiabetic group (pressure side) shows net NOS immunoreactions of $13.1 \%$ of total area (1631705). 


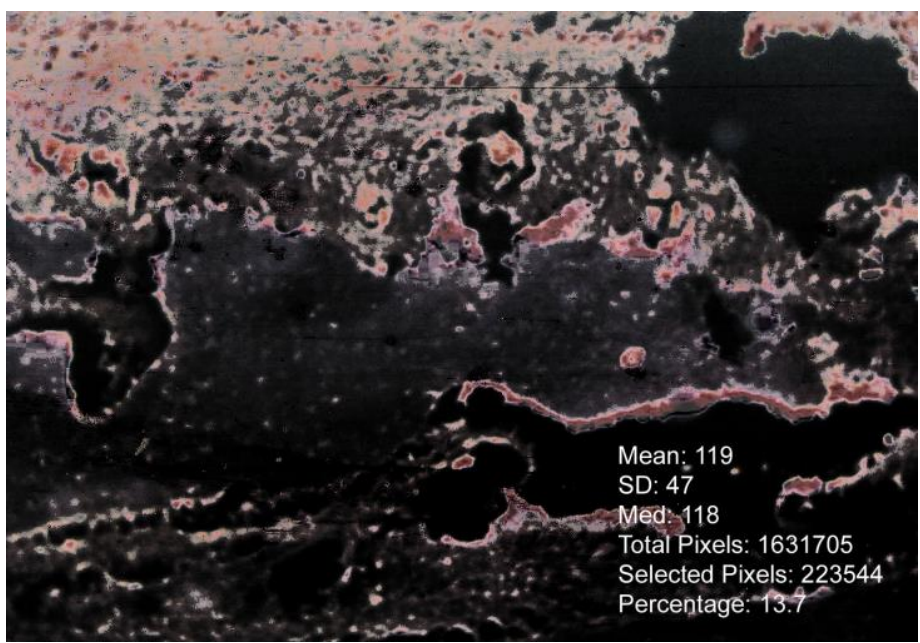

Fig. (10) : digital image analysis of group diabetic (pressure side) showing net NOS immunoreactions of $13.7 \%$ of total area (1631705).

\section{DISCUSSION}

\section{Bone resorption}

As regard to the numbers of osteoclast which revealed from $H \& E$ stain, the diabetic group showed greater numbers of osteoclast in their lacuna indicating more bone resorption compared to non diabetic rats. The bone surface had multideep concavities of Howship's lacuna. On the other hand, they were so shallow lacuna in non diabetic group. Disfigurements were shown in the periodontal ligament fibers, angiopathies of blood vessels and some minute resorption in the cementum in the diabetic rats. These features support the results of many investigators Holtgrave \& Donath ${ }^{(1)}$, Bench et al ${ }^{(2)}$.

The expression of Nitric oxide in the periodontal ligament, bone surfaces and in the blood vessels wall depicts the presence of intense immunopositive reactions of NO to the mechanical stress of orthodontic tooth movement (OTM). However this expression was less intense in the healthy group. These finding explain the greater resorption in diabetic group and support the conclusion of Akin et al ${ }^{(19)}$. 
Other support for the quantity measure of nitric oxide was approved by computerized digital image, which illustrated highly percentage of NO reaction in the diabetic group $(13.7 \%)$ and its value was $13.1 \%$ in healthy group.

\section{$\underline{\text { Bone apposition }}$}

The findings of this study revealed active bone formation in the tension side. However, this bone formation was clear in the form of osteoid tissue with active osteobalstic cells in the healthy group. On the other group, this bone activity was limited, the collagen syntheses was scanty and low density compared to the other non diabetic group. This result of low regeneration activity was cleared in trichrome stain which showed the difference between the two groups in collagen formation. These features were in agreement with Oliver $\&$ Tervonen ${ }^{(20)}$.

In spite of expression of the NO that was more in the pressure side of the diabetic group, it was founded with low reaction in the tension side. Moreover, its reaction was higher in healthy group. This explain the higher bone activity in healthy group compared to the diabetic one. The computerized digital image illustrated a greater percentage of immunopositive reaction in the tension side of the non diabetic group. Depending on the results of this study, the diabetic rats showed greater bone resorption at the pressure side and retarded bone deposition at the tension side compared to the healthy rats. Hence, orthodontist should consider these results by using a very light force, increasing the gaps between visits, planning to decrease the range of tooth movement, monitoring the oral hygiene and sharing with patients physicians for controlling diabetes during orthodontic managements.

1- Thanks to Abo El-Naga AM Associate Professor, Zoology Department, Faculty of Science, Mansoura University, Egypt. for her brotherly concern and supervision of rats during this study.

2- Thanks also, extended to Ahmed Fouda, demonstrator of Orthodontics. for his photographic help and care to share in this study.

\section{REFERENCES}

1. Holtgrave EA, Donath K : Periodontal reactions to orthodontic forces in the diabetic metabolic state. Fortschr Kieferorthop 1989; 50: 326 - 337.

2. Bensch L., Braem M. and Willems G.: Orthodontic considerations in the diabetic patient. Semin Orthod, 2004; 10: 252-258. 
3. Klein-Nulend J, Helfrich MH, Sterck JGH, Macpherson H, Joldersma M, and Ralston SH.: Nitric Oxide response to shear stress by human bone cells cultures is endothelial. Biochem Biophys Res Comm, 1998; 250: 108-114.

4. Zaman G, Pitsillides AA, Rawlinson Sc, Suswillo RF, Mosley JR, Cheng MZ, et al.: Mechanical stress stimulate nitric oxide production by rapid activation of nitric oxide synthase in osteocytes. J Bone Miner Res, 1999; 14: 1123-31.

5. Van t Hof RJ, Ralston SH.: Nitric oxide in bone. Immunology, 2001;103: 255-61.

6. Shirazi M, Nilforoushan D, Alghasi H, Dehpour AR : The role of nitric oxide in orthodontic tooth movement in rats. Angle Orthod, 2002; $72: 211-5$.

7. Wennstrom JL, Lindhe J, Sinclair F, Thilander B: Some periodontal tissue reactions to orthodontic tooth movement in monkeys. J Clin Periodont,1987; $14: 121-9$.

8. Kehoe MJ, Cohen SM, Zarrinnia K, Cowan A: The effect of acetaminophen, ibuprofen, and misoprostol on prostaglandin E 2 synthesis and the degree of orthodontic tooth movement. Angle Orthod, 1996; 66:339-50.

9. Kobayashi Y, Tagaki H, Sakai H, Hashimoto F and MAtaki S, : Effects of local administration of osteocalsin on experimental tooth movement. Angle Orthod, 1998;68 : 259-60.

10. Keigo H., Kaoru I., Kotaro M., Hisashi S. and Hideo M.: Involvement of nitric oxide in orthodontic tooth movement in rats. Am J Orthod Dentofacial Orthop, 2002; 122: 306-309.

11. Collin Dollery : Therapeutic drugs. (ed 2) 1999 vol 1: p G53-G56.

12. Turner N, White $\mathrm{P}$ : Effect of streotozotocin-induced diabetes on vascular reactivity in genetically hyperinsulinaemic obese Zucker rats. J Cardiovasc Pharmacol, 1996; 27 : 884-890

13. Ahmed A EL-Bialy: The effect gingival fiberotomy versus local use of vitamin D on the rate of orthodontic tooth movement. Ph D Thesis, 1994; Mansoura Faculty of Dentistry: Egypt.

14. Akin E., Gurton A.U. and Olmez H.: Effect of nitric oxide in orthodontic tooth movement in rats. Am J Orthod Dentofacial Orthop, 2004; 126: 608-14.

15. Dury RA, Wallington EA : Carleton's Histological Techniques. ed. 5, Oxford, New York, Toronto.1980; 303-350. 
16. Kiernan JA : Histological and Histochemical Methods, theory and practice. Ed 2, Canada press, 1990; 186-199.

17. Kammerer U, Kapp M, Gassel AM, Richter T, Tank C, Dietl J, Ruck P : A new rapid immunohistochemical staining technique using the En vision antibody complex. J Histochem Cytochem, 2001; 49: 623 - 630.

18. Sabattini E, Bisgaard K, Ascani S, Poggi S, Piccioli M, Ceccarelli C, Pieri F, Fraternali-Orcioni G, pileri SA : The En Vision ++ system: a new immunohistochemical method for diagnostics and research Critical comparison with the APAAP, Chem Mate, CSA, LABC, and SABC techniques. J Clin Pathol, 1998; 51: $506-511$.

19. Dahab G, Kheriza M, El-Beltagi H, Fouda AM, Sharaf EL-Din O : Digital quantification of fibrosis in liver biobsy sections : Description of a new method by Photoshop software. J Gastroenterol Hepatol 2004; 19 (1) : 78-85.

20. Oliver RC, Tervonen $\mathrm{T}$ : Periodontitis and tooth loss: comparing diabetics with the general population. J Am Dent Assoc, 1993; 124 :71-76. 